\title{
Laparoscopic Right Adrenalectomy for a Large Adrenal Tumor Combined with in situ Ablation of a Hepatic Nodule in a Cirrhotic Patient
}

\author{
Mirela Patricia Sîrbu Boeți ${ }^{1,2}$, Andrei Marius Baicu ${ }^{2}$, Mihai Toma ${ }^{1}$, Vlad Herlea ${ }^{1,3}$, Laura lliescu ${ }^{1,2}$
}

'Fundeni Clinical Institute, Bucharest, Romania

2"Carol Davila" University of Medicine and Pharmacy, Bucharest, Romania

"'Titu Maiorescu" University, Faculty of Medicine, Bucharest, Romania

\author{
Corresponding author: \\ Mirela Patricia Sîrbu Boeți MD, \\ PhD Fundeni Clinical Institute \\ Bucharest, Romania \\ 258 Fundeni Street, District 2 \\ Bucharest, Romania \\ E-mail: patriciaboeti@gmail.com
}

\section{ABSTRACT}

Introduction: Cirrhotic patients are difficult patients to operate on and are generally considered unsuitable for laparoscopy $(1,2)$. We present the clinical case of a cirrhotic patient with comorbidities that was diagnosed with a hepatic nodule and cortisol secreting right adrenal tumor, for which a multidisciplinary team decided to submit him for laparoscopic intervention.

Case report: N.D. is a 59 y.0. male, diagnosed in 2010 with alcoholic Child-Pugh B liver cirrhosis (score 9). In February 2019, a hepatic nodule in segment IV-A $(2 \mathrm{~cm})$ and cortisol secreting right adrenal tumor $(6 / 4 / 4 \mathrm{~cm})$ were found. He is also diagnosed with essential arterial hypertension grade II, mild diastolic dysfunction of the left ventricle, aortic sclerosis, chronic ischemic cardiomyopathy, type 2 diabetes mellitus and chronic gastritis. He had laparoscopic cholecystectomy in 2000. The hepatic nodule in segment IV-A was highly suggestive of hepatocellular carcinoma due to imaging characteristics. Subclinical secretion of cortisol was present (low ACTH $4.18 \mathrm{pg} / \mathrm{ml}$, normal plasmatic cortisol, metanephrine, normetanephrine). AFP, CEA, CA 19-9 were normal. The patient underwent surgery on 27 May 2019. Adhesiolysis, transperitoneal laparoscopic right adrenalectomy and alcoholization of hepatic tumor have been performed. Radiofrequency ablation of the hepatic tumor was preoperatively anticipated but was intraoperatively abandoned in favor of in situ hepatic tumor ablation with percutaneous ethanol injection due to encountered metabolic acidosis and cardiac arrhythmia.

Results: Postoperatively the patient developed hemorrhagic ascites and abdominal wall hematoma that were successfully controlled by conservative measurements. He was discharged on the $15^{\text {th }}$ postoperative day. Histopathology found cortical-adrenal adenoma with clear cells. The patient was completely withdrawn from corticosteroids in October 2019. On October 1st, 2019, transarterial chemoembolization (TACE) with Gelaspon, Lipidol and Doxorubicin was performed to completely ablate the hepatic nodule. After two years the patient had Child-Pugh B cirrhosis (score 7) with no tumor recurrence.

Conclusion: Laparoscopic right adrenalectomy could be safely performed for a right adrenal tumor in a Child-Pugh B cirrhotic patient with other comorbidities but the percutaneous alcoholization of the hepatic nodule under video and ultrasound guidance needed TACE for its complete destruction.

Key words: laparoscopic adrenalectomy, cirrhosis, hepatic nodule ablation, hepatocellular carcinoma, adrenal adenoma, adrenal tumor
Received: 09.04.2021

Accepted: 13.06.2021 


\section{Conflict of interests}

The authors declare no conflict of interests.

\section{Ethical approval}

The case was approved by the Ethics Committee.

\section{REFERENCES}

1. Zaidi N, Berber E. Endocrine Surgery in Cirrhotic Patients. In: Eghtesad B., Fung J. (eds) Surgical Procedures on the Cirrhotic Patient. Springer; 2017. p. 285-300.

2. Niinobu T, Yamashita S, Nakagawa S, Amano M, Higaki N, Hayashida $\mathrm{H}$, et al. Laparoscopic adrenalectomy for the adrenal gland metastasis from hepatocellular carcinoma. Gan To Kagaku Ryoho. 2006;33(12):1765-7. Japanese 